\title{
Diagnostic value of four-dimensional ultrasonography with STIC combined with two-dimensional ultrasonography for fetal cardiac malformation and chromosomal abnormalities in early pregnancy
}

\author{
HUIDONG LI, FANGHUA PENG, CHAO WU, DEXUAN KONG, QI ZHANG and ZHIKUN ZHANG \\ Department of Ultrasound, Tianjin Central Hospital of Gynecology and Obstetrics, Tianjin 300100, P.R. China
}

Received March 15, 2019; Accepted July 26, 2019

DOI: $10.3892 /$ etm.2019.8325

\begin{abstract}
Diagnostic value of four-dimensional ultrasonography with spatio-temporal image correlation (STIC) technique combined with two-dimensional ultrasonography for fetal cardiac malformation and chromosomal abnormalities in early pregnancy was investigated. Medical data of 178 pregnant women enrolled in Tianjin Central Hospital of Gynecology and Obstetrics for screening of fetal cardiac malformations and chromosomal abnormalities from January 2014 to March 2016 were collected. According to the results of the pregnant women's biopsy and the results of labor induction and delivery, 95 fetuses were diagnosed with chromosomal abnormalities and cardiac malformations, 40 fetuses with cardiac malformations and no chromosomal abnormalities, and 43 fetuses with neither cardiac malformations nor chromosomal abnormalities. Fetal cardiac malformations and chromosomal abnormalities were diagnosed by three methods: single two-dimensional ultrasonography, single four-dimensional ultrasonography with STIC, and a combination of two-dimensional ultrasonography and four-dimensional ultrasonography with STIC. The two-dimensional ultrasonography diagnosed 97 cases of fetal cardiac malformation and 61 cases of chromosomal abnormalities, four-dimensional ultrasonography with STIC diagnosed 122 cases of fetal cardiac malformation and 81 cases of chromosomal abnormalities. The combination of two-dimensional ultrasonography and four-dimensional ultrasonography diagnosed 130 cases of fetal cardiac malformation and 90 cases of chromosomal abnormalities. The sensitivities of fourdimensional ultrasonography with STIC and the combination of two-dimensional ultrasonography and four-dimensional ultrasonography were significantly higher than that of single examination by two-dimensional ultrasonography $(\mathrm{P}<0.05)$,
\end{abstract}

Correspondence to: Dr Zhikun Zhang, Department of Ultrasound, Tianjin Central Hospital of Gynecology and Obstetrics, 156 Nankai Sanma Road, Tianjin 300100, P.R. China

E-mail:ktq4eb@163.com

Key words: two-dimensional ultrasonography, four-dimensional ultrasonography with STIC technique, combined diagnosis, cardiac malformation, diagnostic value, chromosomal abnormality while the diagnostic sensitivities by four-dimensional ultrasonography with STIC and the combined diagnosis were not statistically significant $(\mathrm{P}>0.05)$. The diagnostic accordance rates of the single four-dimensional ultrasonography and the combined diagnosis for fetal cardiac malformations and chromosomal abnormalities were not much different $(\mathrm{P}>0.05)$, but both were greatly higher than that of single two-dimensional ultrasonography diagnosis $(\mathrm{P}<0.05)$. Thus, a combination of four-dimensional ultrasonography with STIC and twodimensional ultrasonography is recommended for screening fetal defects.

\section{Introduction}

Fetal cardiac malformation, a congenital heart disease that damages the life and health of the fetus, is one of the main causes of fetal death (1). Therefore, screening for heart disease in pregnant women during early pregnancy is of great clinical significance, and plays an important role in the birth rate and birth quality of fetus (2). Various factors could cause fetal cardiac malformations in fetus, and many of them are related to chromosomal variation, which, even at a small degree, may cause severe structural abnormalities in the fetal heart, thereby inducing fetal cardiac malformation $(3,4)$. Two-dimensional ultrasonography has been widely used as a basic examination method in the diagnosis of fetal cardiac malformation (5). The screening for chromosomal abnormalities is mainly carried out by serum detection in the second trimester; further diagnosis by amniocentesis or ultrasonography will be performed if abnormalities are found, and the pregnancy is terminated once chromosomal abnormalities are confirmed (6). Although amniocentesis has a high diagnostic accuracy, the action of puncture or termination of pregnancy in the second trimester will cause greater harm to pregnant women, so great clinical significance is attached to finding a non-invasive method with a high accurate rate of chromosomal abnormality screening (7).

Ultrasound testing, as the most commonly used non-invasive method of prenatal examination, plays an important role in fetal cardiac malformation and chromosome screening $(8,9)$. Two-dimensional ultrasonography is commonly applied in the screening of fetal cardiac malformation and chromosomal abnormalities, but it has defect due to the limitation that its planar image can only display one side of the fetus and can only detect limited indexes $(10,11)$. Compared with conventional 
two-dimensional ultrasonography diagnosis, four-dimensional ultrasonography with spatio-temporal image correlation (STIC) technology can offer a more comprehensive and intuitive observation of the fetal heart dynamically or statically (12). Although the current four-dimensional ultrasonography with STIC technique has been reported in some studies on the diagnosis of fetal cardiac malformation, few studies have explored its application in chromosome screening. Therefore, to compare with the conventional two-dimensional ultrasonography, this study explored the diagnostic value of four-dimensional ultrasonography with STIC combined with two-dimensional ultrasonography in screening fetal cardiac malformation and chromosomal abnormalities in early pregnancy.

\section{Materials and methods}

General information. A retrospective analysis was carried out on the medical data of 178 high-risk pregnant women (average age of $24.71 \pm 3.64$ years) with abnormal fetal indications who enrolled in Tianjin Central Hospital of Gynecology and Obstetrics (Tianjin, China) for screening of fetal cardiac malformations and chromosomal abnormalities from January 2014 to March 2016. According to the results of the pregnant women's biopsy and the results of labor induction and delivery, 95 fetuses were diagnosed with chromosomal abnormalities and cardiac malformations, 40 fetuses with cardiac malformations and no chromosomal abnormalities, and 43 fetuses with neither cardiac malformations nor chromosomal abnormalities. Inclusion criteria: high-risk pregnant women with fetal heart malformations or chromosomal abnormalities, who was $<3$ months pregnant and received prenatal care in Tianjin Central Hospital of Gynecology and Obstetrics. Exclusion criteria: pregnant women with a history of congenital heart disease; with severe liver and kidney dysfunction; with severe infections; with puncture contraindications; with cognitive or communication disorders and pregnant women who refused the biopsy.

This study was approved by the Ethics Committee of Tianjin Central Hospital of Gynecology and Obstetrics. All pregnant women and their families agreed to participate in the study and signed an informed consent. The basic patient information is shown in Table I.

\section{Inspection method}

Two-dimensional ultrasonography. All the pregnant women were screened by two-dimensional ultrasonography, at a probe frequency of 5 to $7 \mathrm{MHz}$ using the Voluson E8 model of color ultrasonic diagnostic equipment by GE Healthcare. During the two-dimensional ultrasonography examination, all patients were kept in the supine position to expose the abdomen for observing the fetal heart structure. Various parameters of the fetal heart appearance, ventricular septum, pulmonary aorta, mitral valve and other regions were recorded to detect abnormal ultrasound findings, and chromosomal abnormalities were diagnosed based on fetal soft indicators (including ventricular highlights, strong bowel echo, single umbilical artery, renal pelvic dilatation, choroid plexus cyst and tricuspid regurgitation).

Four-dimensional ultrasonography. Voluson E8 model of color ultrasonic diagnostic equipment by GE Healthcare was
Table I. Patient characteristics [n (\%)].

\begin{tabular}{lc}
\hline Factor & $\begin{array}{c}\text { Pregnant women } \\
(\mathrm{n}=178)\end{array}$ \\
\hline Age $($ years $)$ & $105(58.99)$ \\
$\geq 24$ & $73(41.01)$ \\
$<24$ & \\
BMI $(\mathrm{kg} / \mathrm{m})$ & $101(56.74)$ \\
$\geq 21$ & $77(43.26)$ \\
$<21$ & \\
Educational level & $121(67.98)$ \\
$\geq$ Junior middle school & $57(32.02)$ \\
$<$ Junior middle school & \\
Blood coagulation & $27.97 \pm 2.81$ \\
APTT $($ sec $)$ & $11.77 \pm 1.11$ \\
PT $($ sec $)$ & $3.41 \pm 0.25$ \\
FIB $(\mathrm{g} / \mathrm{l})$ & $14.23 \pm 1.41$ \\
TT $(\mathrm{sec})$ & $25.39 \pm 3.29$ \\
Average pregnancy time & \\
Liver function index & $72.61 \pm 2.55$ \\
Total protein $(\mathrm{g} / \mathrm{l})$ & $28.52 \pm 4.77$ \\
Alanine transferase $(\mu \mathrm{mol} / \mathrm{l})$ & $11.56 \pm 2.09$ \\
Total bilirubin $(\mu \mathrm{mol} / \mathrm{l})$ & \\
Renal function index & $68.54 \pm 4.37$ \\
Creatin $(\mu$ mol/l) & $5.29 \pm 0.74$ \\
Serum urea $(\mu \mathrm{mol} / \mathrm{l})$ & $288.65 \pm 12.41$ \\
Uric acid $(\mu$ mol/l) & \\
\hline
\end{tabular}

used. The frequency of four-dimensional ultrasonic probe (RAB4-8-D) was 2.5-7.0 MHz, and the QLAB software was equipped with four-dimensional ultrasonography to support STIC technology. All women were in supine position to expose the abdomen, and then the four-dimensional ultrasonography was used to collect images of the four-chamber view of the fetal heart or the long axis section of the aortic arch, and to scan the fetal heart, large blood vessels and neck. The four-chamber heart, the left ventricular outflow tract, the right ventricular outflow tract, the three-vessel tracheal plane, the short-axis view of the heart, the long axis plane of the aorta, the long axis plane of the pulmonary artery, and the longitudinal section of the superior and inferior vena cava are displayed to determine the fetal heart malformation. The STIC technique was performed to acquire a collection of the relevant volume data. Two ultrasound doctors without knowledge of the two-dimensional diagnosis results independently analyzed the data. This diagnosis was limited to the diagnosis made by the four-dimensional STIC technique, and no diagnostic report was issued. Chromosome abnormal soft index detection criteria: i) neck transparent (NT) thickening, NT in 11 to $13+6$ weeks of pregnancy detected by ultrasound is greater than the 95th percentile; ii) ultrasound median sagittal section nasal bone strong echo suggests nasal bone dysplasia or loss; iii) intestinal strong echo and bone tissue strength are equivalent to intestinal echo enhancement; iv) single 
Table II. Diagnosis of fetal cardiac malformation.

\begin{tabular}{|c|c|c|c|c|c|c|}
\hline \multirow[b]{2}{*}{ Diagnostic result } & \multicolumn{2}{|c|}{$\begin{array}{l}\text { Two-dimensional } \\
\text { ultrasonography }\end{array}$} & \multicolumn{2}{|c|}{$\begin{array}{l}\text { Four-dimensional } \\
\text { ultrasonography }\end{array}$} & \multicolumn{2}{|c|}{ Combined diagnosis } \\
\hline & $\begin{array}{c}\text { Pathology } \\
\text { (malformation) }\end{array}$ & $\begin{array}{c}\text { Pathology } \\
\text { (normal) }\end{array}$ & $\begin{array}{c}\text { Pathology } \\
\text { (malformation) }\end{array}$ & $\begin{array}{c}\text { Pathology } \\
\text { (normal) }\end{array}$ & $\begin{array}{c}\text { Pathology } \\
\text { (malformation) }\end{array}$ & $\begin{array}{c}\text { Pathology } \\
\text { (normal) }\end{array}$ \\
\hline Diagnosis (malformation) & 97 & 17 & 122 & 13 & 130 & 13 \\
\hline Diagnosis (normal) & 38 & 26 & 13 & 30 & 13 & 30 \\
\hline Total number & 135 & 43 & 135 & 43 & 135 & 43 \\
\hline
\end{tabular}

umbilical artery; v) choroidal echo shows ovarian cyst in elliptical or round cystic structure; vi) ventricular strong echo flare; vii) unilateral or bilateral lateral ventricle width up to $10-15 \mathrm{~mm}$ suggests lateral ventricle dilatation; viii) renal pelvis separation; and ix) the length diameter of femur is less than 2 standard deviations in normal gestational age.

Observation indicators. According to the labor induction or diagnosis of the newborns, the sensitivity, specificity, diagnostic accordance rate, positive predictive value and negative predictive value were compared between the three examination methods: the single two-dimensional ultrasonography, the single four-dimensional ultrasonography with STIC techniques, and the combination of two-dimensional ultrasonography and four-dimensional ultrasonography with STIC. According to the diagnosis of chromosomal variation by amniocentesis, the screening results of chromosomal abnormalities of the three examination methods were compared.

Statistical analysis. The SPSS 20.0 software (IBM Corp.) was used for the analysis of the experimental data. The Chi-square test was used for enumeration data, and the $t$-test was used for the measurement data. $\mathrm{P}<0.05$ was considered to indicate a statistically significant difference. Results are presented as percentage.

\section{Results}

Diagnostic value of two-dimensional ultrasonography, four-dimensional ultrasonography with STIC and the combined diagnosis for cardiac malformation. Ninety-seven cases of fetal cardiac malformation were diagnosed by two-dimensional ultrasonography, 122 by four-dimensional ultrasonography with STIC, and 130 by the combination of two-dimensional ultrasonography and four-dimensional ultrasonography with STIC (Table II). The sensitivity, specificity, diagnostic accordance rate, negative predictive value, and the positive predictive values of the two-dimensional ultrasonography diagnosis were $71.85,60.47,69.10,40.63$, and $85.09 \%$, respectively; those of the four-dimensional ultrasonography with STIC diagnosis were 90.37, 69.77, 85.39, 69.77, and $90.37 \%$, respectively; those of the combined diagnosis were $96.30,51.16,85.39,81.48$ and $86.09 \%$, respectively. The sensitivities of four-dimensional ultrasonography with STIC and the combined diagnosis were statistically much higher than that of two-dimensional ultrasonography diagnosis $(\mathrm{P}<0.05)$, and the sensitivity of the combined diagnosis was slightly higher than that of four-dimensional ultrasonography with STIC, but the difference was not statistically significant $(\mathrm{P}>0.05)$. The negative predictive value of combined detection was significantly higher than that of single two-dimensional ultrasonography and single four-dimensional ultrasonography with STIC, and the difference was statistically significant $(\mathrm{P}<0.05)$. The negative predictive value of four-dimensional ultrasonography with STIC was also significantly higher than that of twodimensional ultrasonography $(\mathrm{P}<0.05)$. The specificity and positive predictive value of four-dimensional ultrasonography with STIC were higher than those of single two-dimensional ultrasonography and the combined diagnosis, but the differences were not statistically significant $(\mathrm{P}>0.05)$. No significant difference was detected in the diagnostic accordance rate between four-dimensional ultrasonography diagnosis and the combined diagnosis $(\mathrm{P}>0.05)$, but both were statistically much higher than the diagnostic accordance rate of the single twodimensional ultrasonography $(\mathrm{P}<0.05)$ (Table III).

Performance analysis of the three examination methods for diagnosis of fetal chromosomal abnormalities. Sixty-one cases of fetal chromosomal abnormalities were diagnosed by two-dimensional ultrasonography, 81 by four-dimensional ultrasonography with STIC, and 90 by the combined diagnosis (Table IV). The sensitivity, specificity, diagnostic accordance rate, negative predictive value, and the positive predictive value of the two-dimensional ultrasonography diagnosis were $64.21,60.24,62.36,59.52$, and $64.89 \%$, respectively; those of the four-dimensional ultrasonography with STIC diagnosis were $85.26,73.49,79.78,81.33$, and $78.64 \%$, respectively; and those of combined diagnosis were 94.74, 63.86, 80.34, 91.38, and $75.00 \%$, respectively. The sensitivity of combined diagnosis was significantly higher than that of the single two-dimensional ultrasonography diagnosis and the single four-dimensional ultrasonography with STIC, and the difference was statistically significant $(\mathrm{P}<0.05)$; while the sensitivity of four-dimensional ultrasonography with STIC was statistically much higher than that of two-dimensional ultrasonography diagnosis $(\mathrm{P}<0.05)$. The negative predictive value of the combined diagnosis was statistically much higher than that of the two-dimensional ultrasonography and the four-dimensional ultrasonography with STIC $(\mathrm{P}<0.05)$; and the negative predictive value of the single four-dimensional ultrasonography with STIC was also significantly higher than that of the two-dimensional ultrasonography $(\mathrm{P}<0.05)$. No significant difference in specificity and positive predictive value was shown between the three 
Table III. Performance analysis of the three examination methods [n (\%)].

\begin{tabular}{|c|c|c|c|c|c|}
\hline Diagnostic value & $\begin{array}{l}\text { Two-dimensional } \\
\text { ultrasonography }\end{array}$ & $\begin{array}{c}\text { Four-dimensional } \\
\text { ultrasonography } \\
\text { with STIC }\end{array}$ & $\begin{array}{l}\text { Combined } \\
\text { diagnosis }\end{array}$ & $\chi^{2}$ value & P-value \\
\hline Sensitivity & $97(71.85)$ & $122(90.37)$ & $130(96.30)$ & 36.84 & $<0.001$ \\
\hline Specificity & $26(60.47)$ & $30(69.76)$ & $22(51.16)$ & 3.113 & 0.210 \\
\hline Diagnostic accordance rate & $123(69.10)$ & $152(85.39)$ & $152(85.39)$ & 19.66 & $<0.001$ \\
\hline Negative predictive value & $43(40.63)$ & $30(69.77)$ & $22(81.48)$ & 16.51 & $<0.050$ \\
\hline Positive predictive value & $97(85.09)$ & $122(90.37)$ & $130(86.09)$ & 1.843 & 0.398 \\
\hline
\end{tabular}

STIC, spatio-temporal image correlation.

Table IV. Diagnosis of fetal chromosomal abnormalities.

\begin{tabular}{|c|c|c|c|c|c|c|}
\hline \multirow[b]{2}{*}{ Diagnostic result } & \multicolumn{2}{|c|}{$\begin{array}{l}\text { Two-dimensional } \\
\text { ultrasonography }\end{array}$} & \multicolumn{2}{|c|}{$\begin{array}{c}\text { Four-dimensional } \\
\text { ultrasonography }\end{array}$} & \multicolumn{2}{|c|}{ Combined diagnosis } \\
\hline & $\begin{array}{c}\text { Pathology } \\
\text { (malformation) }\end{array}$ & $\begin{array}{c}\text { Pathology } \\
\text { (normal) }\end{array}$ & $\begin{array}{c}\text { Pathology } \\
\text { (malformation) }\end{array}$ & $\begin{array}{l}\text { Pathology } \\
\text { (normal) }\end{array}$ & $\begin{array}{c}\text { Pathology } \\
\text { (malformation) }\end{array}$ & $\begin{array}{c}\text { Pathology } \\
\text { (normal) }\end{array}$ \\
\hline Diagnosis (malformation) & 61 & 33 & 81 & 22 & 90 & 30 \\
\hline Diagnosis (normal) & 34 & 50 & 14 & 61 & 5 & 53 \\
\hline Total number & 95 & 83 & 95 & 83 & 95 & 83 \\
\hline
\end{tabular}

Table V. Performance analysis of the three examination methods [n (\%)].

\begin{tabular}{|c|c|c|c|c|c|}
\hline Diagnostic value & $\begin{array}{l}\text { Two-dimensional } \\
\text { ultrasonography }\end{array}$ & $\begin{array}{c}\text { Four-dimensional } \\
\text { ultrasonography } \\
\text { with STIC }\end{array}$ & $\begin{array}{l}\text { Combined } \\
\text { diagnosis }\end{array}$ & $\chi^{2}$ value & P-value \\
\hline Diagnostic value & $61(64.21)$ & $81(85.26)$ & $90(94.74)$ & 30.64 & $<0.050$ \\
\hline Sensitivity & $50(60.24)$ & $61(73.49)$ & $53(63.86)$ & 3.465 & 0.177 \\
\hline Specificity & $111(62.36)$ & $142(79.78)$ & $143(80.34)$ & 19.41 & $<0.050$ \\
\hline Diagnostic accordance rate & $50(59.52)$ & $61(81.33)$ & $53(91.38)$ & 20.92 & $<0.050$ \\
\hline Negative predictive value & $61(64.89)$ & $81(78.64)$ & $90(75.00)$ & 5.057 & 0.080 \\
\hline
\end{tabular}

STIC, spatio-temporal image correlation.

methods of diagnosis $(\mathrm{P}>0.05)$; the diagnostic accordance rates in the four-dimensional ultrasonography with STIC and the combined diagnosis were not statistically significant $(\mathrm{P}>0.05)$, but both were significantly higher than the diagnostic accordance rate of two-dimensional ultrasonography diagnosis, and the difference was statistically significant $(\mathrm{P}<0.05)$ (Table $\mathrm{V})$.

\section{Discussion}

Ultrasound testing is the most commonly used test for prenatal examination, and plays an important role in screening fetal cardiac malformation and chromosomal abnormalities $(13,14)$. However, in the screening of fetal chromosomal abnormalities, prenatal invasive diagnosis such as amniocentesis which remains the gold standard for diagnosing abnormalities in fetal chromosomes, is a traumatic operation with high risk of infection and abortion that makes pregnant women unwilling to accept it (15). In spite of the wide application of ultrasound in fetal cardiac malformation, few studies are reported on the screening of fetal cardiac malformation by four-dimensional ultrasonography with STIC technology. Search for a non-invasive, easy-to-accept and effective screening method for chromosomal abnormalities is urgent, therefore, screening of fetal chromosomal abnormalities when screening fetal cardiac malformations by four-dimensional ultrasonography with STIC was conducted.

In this study, the performance analysis of the three examination methods for diagnosis of fetal cardiac malformation was performed. According to the results, the sensitivities of single four-dimensional ultrasonography with STIC and the 
combined diagnosis were significantly higher than that of single two-dimensional ultrasonography $(\mathrm{P}<0.05)$, and the sensitivity of the combined diagnosis was slightly higher than that of the single four-dimensional ultrasonography with STIC, but the difference was not statistically significant $(\mathrm{P}>0.05)$; the negative predictive value of the combined diagnosis was significantly higher than that of the single two-dimensional ultrasonography and single four-dimensional ultrasonography with STIC $(\mathrm{P}<0.05)$, and the negative predictive value of the single four-dimensional ultrasonography with STIC was also significantly higher than that of the single two-dimensional ultrasonography diagnosis $(\mathrm{P}<0.05)$; no significant difference in the specificity and the positive predictive value was detected between the three examination methods ( $P>0.05)$; no significant difference presented in the diagnostic accordance rates between the four-dimensional ultrasonography diagnosis and the combined diagnosis $(\mathrm{P}>0.05)$, but both were significantly higher than the diagnostic accordance rate of the two-dimensional ultrasonography diagnosis $(\mathrm{P}<0.05)$. Although capable of making analysis of the fetal heart shape and size, the twodimensional ultrasonography section can hardly show a clear view for the complex internal structure of the fetus (16). The four-dimensional STIC imaging can not only offer the fourdimensional information of the fetal heart through volumetric scanning to ensure a more stereoscopical observation, but also adjust section for different pregnant woman to improve the diagnostic accuracy for cardiac malformation $(17,18)$.

A previous study stated that four-dimensional ultrasonography with STIC has a higher diagnostic rate than traditional two-dimensional ultrasonography in the diagnosis of fetal cardiac malformation, which is consistent with the result of this study (17). The study doubted that the imaging feature of STIC would impact the fetal chromosomal abnormality screening, but no such impact has been reported so far. Therefore, our study made comparison of the three examination methods in the diagnosis of fetal chromosomal abnormalities. According the results of chromosomal abnormality diagnosis, the sensitivities of the combined diagnosis and the single four-dimensional ultrasonography with STIC were much higher than that in the single two-dimensional ultrasonography $(\mathrm{P}<0.05)$, and the sensitivity of the combined diagnosis was much higher than that in the single four-dimensional ultrasonography with STIC $(\mathrm{P}>0.05)$. The negative predictive value of the combined diagnosis was significantly higher than that of the single four-dimensional ultrasonography with STIC $(\mathrm{P}<0.05)$ which had much higher negative predictive value than the single two-dimensional ultrasonography $(\mathrm{P}<0.05)$. No significant difference in specificity and positive predictive value was shown between the three methods of diagnosis $(\mathrm{P}>0.05)$. The diagnostic accordance rates in the four-dimensional ultrasonography with STIC and the combined diagnosis were not statistically significant $(\mathrm{P}>0.05)$, but both were significantly higher than the diagnostic accordance rate of two-dimensional ultrasonography diagnosis $(\mathrm{P}<0.05)$. At present, there are a large amount of common soft indicators in ultrasonography for chromosomal abnormalities in early pregnancy, such as cardiac malformation, NT thickening, nasal bone truncation and tricuspid regurgitation (19). Specific soft indicators were not given in this study, but fetal chromosomal abnormality was diagnosed according to the soft indicators. Although the appearance of ultrasound soft indicators does not mean that the fetus must have chromosomal abnormalities, it suggests that the fetus has some unknown risk and is an important suggestion of chromosomal abnormalities (20). A previous study (21) showed that the sensitivity of screening for chromosomal abnormalities with a single soft index is lower than screening with multiple soft indicators. Further research on the comparison of sensitivity need to be performed.

In summary, the four-dimensional ultrasonography with STIC has a higher diagnostic value and less injury to the fetus in the diagnosis of fetal malformations and chromosomal abnormalities in pregnant women than the conventional twodimensional ultrasonography. Additionally, the combination of four-dimensional ultrasonography with STIC and twodimensional ultrasonography is recommended in abnormality screening, since the combined diagnosis had a higher diagnostic value than the single four-dimensional ultrasonography with STIC diagnosis. There may be some children with both cardiac malformation and chromosomal abnormalities. Therefore, application of ultrasound in fetal cardiac malformation and chromosomal abnormality screening is still required.

\section{Acknowledgements}

Not applicable.

\section{Funding}

This study was supported by Tianjin Science and Technology Commission key project (no. 15ZCZDSY00990) and Tianjin Health Bureau Science and Technology fund (no. 2014KZ071).

\section{Availability of data and materials}

The datasets used and/or analyzed during the present study are available from the corresponding author on reasonable request.

\section{Authors' contributions}

HL wrote the manuscript and was also involved in the conception of the study. FP analyzed and interpreted the patient data. CW performed the experiment and designed the study. DK and QZ were responsible for the analysis and discussion of the data. ZZ designed the methods. All authors read and approved the final manuscript.

\section{Ethics approval and consent to participate}

This study was approved by the Ethics Committee of Tianjin Central Hospital of Gynecology and Obstetrics (Tianjin, China). All pregnant women and their families agreed to participate in the study and signed an informed consent.

\section{Patient consent for publication}

Not applicable.

\section{Competing interests}

The authors declare that they have no competing interests. 


\section{References}

1. Chen KB, Gu Q, Xia T, Lu X and Zhang ZD: Three-vessel-trachea view in the diagnosis of fetal cardiac great vessel malformation. $\mathrm{J}$ Biol Regul Homeost Agents 32: 351-355, 2018.

2. Florjański J, Fuchs T, Zimmer M, Homola W, Pomorski M and Blok D: The role of ductus venosus Doppler flow in the diagnosis of chromosomal abnormalities during the first trimester of pregnancy. Adv Clin Exp Med 22: 395-401, 2013.

3. Picone O, Brisset S, Senat MV, Maurin ML, Frydman R and Tachdjian G: Risk of missed diagnosis of 22q11.2 deletion in a fetal cardiac conotruncal malformation when another chromosomal abnormality is detected. J Gynecol Obstet Biol Reprod (Paris) 37: 299-301, 2008 (In French).

4. Roskes EJ, Boughman JA, Schwartz S and Cohen MM: Congenital cardiovascular malformations (CCVM) and structural chromosome abnormalities: A report of 9 cases and literature review. Clin Genet 38: 198-210, 1990.

5. Rayburn WF, Jolley JA and Simpson LL: Advances in ultrasound imaging for congenital malformations during early gestation. Birth Defects Res A Clin Mol Teratol 103: 260-268, 2015.

6. Barik M, Mishra PR and Mohapatra AK: Missing links between genetically inherited molecules in split cord malformation and other anomaly: A bench to bedside approach. J Pediatr Neurosci 13: 46-57, 2018.

7. Bravo-Valenzuela NJ, Peixoto AB and Araujo Júnior E: Prenatal diagnosis of congenital heart disease: A review of current knowledge. Indian Heart J 70: 150-164, 2018.

8. Spencer K, Spencer CE, Power M, Dawson C and Nicolaides KH: Screening for chromosomal abnormalities in the first trimester using ultrasound and maternal serum biochemistry in a one-stop clinic: A review of three years prospective experience. BJOG 110: 281-286, 2003

9. Picazo-Angelin B, Zabala-Argüelles JI, Anderson RH and Sánchez-Quintana D: Anatomy of the normal fetal heart: The basis for understanding fetal echocardiography. Ann Pediatr Cardiol 11: 164-173, 2018.

10. Shimada S, Yamada H, Hoshi N, Kobashi G, Okuyama K, Hanatani K and Fujimoto S: Specific ultrasound findings associated with fetal chromosome abnormalities. Congenit Anom (Kyoto) 49: 61-65, 2009.

11. Brandão P, Soares E, Estevinho C, Freixo M, Portela-Carvalho AS and Ferreira MJ: Skeletal defect at mid-trimester ultrasound scan. J Med Ultrasound 26: 171-173, 2018.
12. Guasina F, Bellussi F, Morganelli G, Salsi G, Pilu G and Simonazzi G: Electronic spatiotemporal image correlation improves four-dimensional fetal echocardiography. Ultrasound Obstet Gynecol 51: 357-360, 2018.

13. Prats P, Ferrer Q, Comas C and Rodríguez I: Is the addition of the ductus venosus useful when screening for aneuploidy and congenital heart disease in fetuses with normal nuchal translucency? Fetal Diagn Ther 32: 138-143, 2012.

14. Grandjean H, Larroque D and Levi S; The Eurofetus Team: Detection of chromosomal abnormalities, an outcome of ultrasound screening. Ann NY Acad Sci 847: 136-140, 1998.

15. Rivas A, Epelman M, Danzer E, Adzick NS and Victoria T: Prenatal MR imaging features of Caroli syndrome in association with autosomal recessive polycystic kidney disease. Radiol Case Rep 14: 265-268, 2018.

16. Venkataswamy C, Gurusamy U and Lakshmi SV: Secondtrimester fetal autopsy: A morphological study with prenatal USG correlations and clinical implications. J Lab Physicians 10: 338-345, 2018.

17. Uittenbogaard LB, Haak MC, Spreeuwenberg MD and van Vugt JM: Fetal cardiac function assessed with fourdimensional ultrasound imaging using spatiotemporal image correlation. Ultrasound Obstet Gynecol 33: 272-281, 2009.

18. Adam S, Coetzee M and Honey EM: Pena-Shokeir syndrome: Current management strategies and palliative care. Appl Clin Genet 11: 111-120, 2018.

19. Beke A, Papp C, Tóth-Pál E, Mezei G, Joó JG, Csaba A and Papp Z: Trisomies and other chromosome abnormalities detected after positive sonographic findings. J Reprod Med 50: 675-691, 2005.

20. Yeo L, Luewan S, Markush D, Gill N and Romero R: Prenatal diagnosis of dextrocardia with complex congenital heart disease using fetal intelligent navigation echocardiography (FINE) and a literature review. Fetal Diagn Ther 43: 304-316, 2018.

21. Mavrides E, Sairam S, Hollis B and Thilaganathan B: Screening for aneuploidy in the first trimester by assessment of blood flow in the ductus venosus. BJOG 109: 1015-1019, 2002.

This work is licensed under a Creative Commons Attribution-NonCommercial-NoDerivatives 4.0 International (CC BY-NC-ND 4.0) License. 